\title{
Outcome Results of Treatment with Selective Internal Radiation Therapy (SIRT) in Patients with Hepatocellular Carcinoma: A Single Center Experience
}

\author{
Jan Pfeiffenberger ${ }^{1,8}$, Tatjana Zimmermann 1,8, Daniel N. Gotthardt ${ }^{1,8}$, Christoph Springfeld ${ }^{2,8}$, \\ Wolfgang Stremmel1,8, Peter Schirmacher ${ }^{3,8}$, Henning Schulze-Bergkamen ${ }^{4,8}$, Arianeb Mehrabi ${ }^{5,8}$, \\ Christoph W. Michalski5,8, Katrin Hoffmann'5,8, Nikolas Kortes ${ }^{6,8}$, Boris Radeleff6,8, \\ Uwe Haberkorn 7,8, Clemens Kratochwil7,8, Karl Heinz Weiss ${ }^{1,7^{*}}$, Carsten Grüllich ${ }^{2,8^{*}}$ \\ ${ }^{1}$ Department of Gastroenterology and Hepatology, University Hospital Heidelberg, Heidelberg, Germany \\ ${ }^{2}$ Department of Medical Oncology, National Center for Tumor Diseases Heidelberg, University Hospital Heidelberg, Heidelberg, \\ Germany \\ ${ }^{3}$ Institute of Pathology, University Hospital Heidelberg, Heidelberg, Germany \\ ${ }^{4}$ Department of Gastroenterology and Oncology, Marien-Hospital, Wesel, Germany \\ ${ }^{5}$ Department of General and Transplant Surgery, University Hospital Heidelberg, Heidelberg, Germany \\ ${ }^{6}$ Department of Diagnostic and Interventional Radiology, University Hospital Heidelberg, Heidelberg, Germany \\ ${ }^{7}$ Department of Nuclear Medicine, University Hospital Heidelberg, Heidelberg, Germany \\ ${ }^{8}$ Liver Cancer Center Heidelberg, University Hospital Heidelberg, Heidelberg, Germany \\ Email: ^Karl-heinz_weiss@med.uni-heidelberg.de, *Carsten.gruellich@med.uni-heidelberg.de
}

How to cite this paper: Pfeiffenberger, J., Zimmermann, T., Gotthardt, D.N., Springfeld, C., Stremmel, W., Schirmacher, P., Schulze-Bergkamen, H., Mehrabi, A., Michalski, C.W., Hoffmann, K., Kortes, N., Radeleff, B., Haberkorn, U., Kratochwil, C., Weiss, K.H. and Grüllich, C. (2017) Outcome Results of Treatment with Selective Internal Radiation Therapy (SIRT) in Patients with Hepatocellular Carcinoma: A Single Center Experience. Journal of Cancer Therapy, 8, 349-359.

https://doi.org/10.4236/jct.2017.84031

Received: March 18, 2017 Accepted: April 25, 2017 Published: April 28, 2017

\begin{abstract}
Background: Hepatocellular carcinoma (HCC) has a poor prognosis. Selective internal radiation therapy (SIRT) with microspheres is a treatment option for HCC. This study aimed to assess safety and survival (OS) in patients with HCC treated with SIRT, to stratify patients with tumor vascularization and analyze the impact of sequential sorafenib treatment. Methods: Thirty-nine patients who received SIRT for HCC between 2010 and 2013 at our center were included in this retrospective analysis. Tumor vascularization was assessed using a combination of MRI, MAA-scintigraphy and angiography. Tumor vascularization was correlated with survival. Subgroups are treated with two commercially available ${ }^{90} Y$-labeled products SIR-Spheres $(n=16)$ and TheraSpheres $(\mathrm{n}=23)$ and sequential therapy with sorafenib compared to SIRT only was analyzed. Results: Adverse events occurred in $49 \%$ of patients with only four grade 3 and no grade 4 event. Median survival for all patients was 12.5 months (95\% CI: 8.7 - 16.3). No significant differences were detectable between Thera Spheres or SIR Spheres. Survival was shorter in patients with low tumor vascularization score (OS: 3.8 months (95\% CI $0-15.0), p=$
\end{abstract}


Copyright $\odot 2017$ by authors and Scientific Research Publishing Inc. This work is licensed under the Creative Commons Attribution International License (CC BY 4.0).

http://creativecommons.org/licenses/by/4.0/
0.043). Survival was longer with sorafenib upon progression after SIRT $(\mathrm{n}=16)$ with an OS of 17.4 months (95\% CI: 12.1 - 22.7) compared to no sorafenib (n = 13; 9.1 months; $95 \%$ CI: $3.0-15.1$ ) or progression upon sorafenib before SIRT ( $\mathrm{n}=10 ; 8.6$ months; 95\% CI: 5.5 - 11.7). Conclusions: SIRT is safe in HCC patients. Tumor vascularization by radiography and scintigraphy may predict survival benefit. Sorafenib is active after SIRT and significantly prolongs survival.

\section{Keywords}

SIRT, Sorafenib, Liver Cancer, Y90

\section{Introduction}

Prognosis and treatment of hepatocellular carcinoma (HCC) is determined by a combination of the function of the non-tumerous liver and stage of tumor disease. This is reflected within the Barcelona Clinic Liver Cancer (BCLC) classification [1]. Currently, standard treatment for stage B disease is transarterial chemoembolisation (TACE) [2]. TACE has been shown to be an effective therapy for local control of HCC [3] that results in survival prolongation [4]. If TACE is technically impossible or if stage $\mathrm{C}$ disease is present, systemic therapy with sorafenib, an anti-angiogenic tyrosine kinase inhibitor (TKI) is an alternative and has also been shown to prolong survival in HCC patients [5]. A further alternative is the relatively new technique of Selective Internal Radiation Therapy (SIRT). SIRT can be conducted in patients who do not have a tumor supplying vessel which could be embolized by TACE. Two different SIRT spheres are currently available: Thera-spheres and SIR-spheres [6]. Previous studies comparing those two spheres in patients with liver metastasis have not found a difference in safety and efficacy [7] [8], but these cohorts did not contain patients with preexisting liver cirrhosis. Tumor control has been demonstrated in HCC patients treated with SIRT, but a survival benefit is less clear since no prospective randomized trials are available and all data are of retrospective nature so far [9]. To enhance the available data on SIRT we retrospectively analyzed 39 consecutive patients receiving SIRT for HCC at our institution. Aim of this study was to analyze the safety of SIRT in this group of patients with high co-morbidity and further to identify clinical and radiographic parameters associated with prognosis after SIRT. Since many HCC patients who are not eligible for TACE receive sorafenib treatment either prior or post SIRT, we further assessed the effect of sorafenib on the prognosis.

\section{Material and Methods}

\subsection{Patients}

We retrospectively reviewed the medical records of all patients who received SIRT for locally advanced HCC at the University Hospital Heidelberg from 
$1 / 1 / 2010$ to $31 / 12 / 2013$. Diagnosis of HCC was based on histology $(n=25 ; 64$, $1 \%)$ or radiological characteristics based on the current guidelines for diagnosis and treatment of HCC. Patients who underwent liver transplantation prior to SIRT were excluded. Patient's charts were reviewed for presence of chronic liver diseases, time of initial HCC diagnosis, other tumor specific therapies prior or after SIRT and survival. Moreover adverse events were recorded. We stratified the occurrence of adverse events by time of onset: Ultra-short-term (day 1 to 4 after intervention), short-term (day 5 to 30 after intervention) and long-term (day 31 to 90 after intervention). The tumor-specific parameters maximum diameter, number of lesions and relationship to the remaining liver were analyzed.

All patients were 18 years or older. The study design was approved, a priori, by the local ethics committee of the University Hospital Heidelberg.

\subsection{SIRT Procedure}

SIRT is an intraarterial way of brachytherapy. Microspheres from resin (SIRSpheres $^{\mathrm{TM}}$ ) or glass (Theraspheres ${ }^{\mathrm{TM}}$ ) loaded with the beta radiation agent Ytrium90 are injected in the hepatic arteriel branches feeding the target lesions. The Spheres spread into the terminal arterioles of the target and emit beta radiation leading to a local radiotherapeutic effect. The blood supply of the tumors mainly derives from the arterial system, while the non-tumoral parenchyma is fed by the portalvenous system to a significant extent. Thus the differences in arterial vascularization between tumor and non-tumoerous liver determines the amount of intentedradiation delivered to the tumor and unintended toxic radiation of the surrounding non-tumerous liver.

In general, a single session bi-lobar treatment was planned. First, a 4-Fr catheter (Sidewinder-S1) was placed in the celiac artery via a transfemoral access in Seldinger technique using local anesthetic. Then a microcatheter (Progreat) was inserted coaxially and advanced to the proper hepatic artery. In cases of anatomic variation with extra-hepatic branches arising from the proper hepatic artery, the lobar arteries were cannulated and the treatment activity was divided to match the individual target volume ratio.

\subsection{Visual Vascularization Score}

Because SIRT is an intraarterial procedure, the hypervascularity of lesions in baseline contrast-images is an important factor to select patients for SIRT. We calculated a "visual vascularization score" (VVS) by contrast-enhanced MRI, MAA-scintigraphy and angiography and correlated them to response to therapy and outcome. We further created a "combined visual vascularisation score" to evaluate if a combined hypervascularization assessment derived from these three standard baseline images predicts SIRT outcome.

An experienced radiologist evaluated the hypervascularity and the margins of target lesions in MRI, MAA-scans and angiographic images taken for SIRT in a blinded retrospective analysis. By investigator assessment, 3 points were given for well defined tumor margins and strong enhancement ("high"), 2 points for 
less good defined tumor margins and/or medium enhancement ("medium") and 1 point for poor defined tumor margins and weak enhancement of the lesions ("low"). The mean of all three image-specific scores created the overall score (1 3 "low"; 4 - 6 "medium", 7 - 9 "high").

\subsection{Statistical Methods}

Statistical analysis was carried out using SPSS $^{\text {Tw }}$ for Windows ${ }^{\text {Tw }}$ Software V15.0 (SPSS, Chicago IL, USA). Survival analysis was calculated using the KaplanMeier method, and differences in survival were analyzed using the log-rank test. Multivariate analysis was carried out using the Cox-regression model. A p-value of $<0.05$ was considered significant.

\section{Results}

\subsection{Patient's Characteristics}

Thirty-nine consecutive patients were included in the study. Median age was 65 (interquartile range 57 - 68) years. 33 patients (85\%) were male. Clinically confirmed liver cirrhosis was present in 36 of the patients (92\%). Of these 34 patients (94\%) had liver function Child-Pugh-score A and two patients (6\%) had Child-Pugh-Score B. Etiology of cirrhosis was chronic hepatitis B in 13 patients (33\%), chronic alcohol abuse in 9 patients $(23 \%)$, chronic hepatitis $\mathrm{C}$ in 8 patients $(20.5 \%)$, cryptogenic in 8 patients $(20.5 \%)$ and Non-alcoholic steatohepatitis (NASH) in one patient (3\%). 38 patients (97\%) were in the "Barcelona Clinic Liver Cancer"-stage B, one patient was in stage C. HCC was proven by biopsy in $25(64 \%)$ of the cases. For detailed patients characteristics see Table 1.

\subsection{SIRT Technique and Outcome}

Two different microspheres are currently available, glass micospheres (TheraSpheres $^{\mathrm{T}}{ }^{\mathrm{Tx}}$ ) and resin micospheres (SIR-Spheres ${ }^{\mathrm{Tw}}$ ) with different diameter, specific activity, and therefore different number of spheres needed for treatment. We used TheraSpheres $^{\mathrm{Tu}}$ in 23 patients (56\%) and SIR Spheres $\odot$ in 16 patients (41\%). Median administered treatment activity in our cohort was 3.0 (1.5 - 4.6) GBq for TheraSpheres $^{\mathrm{Th}}$ and $1.6(0.6-2.0) \mathrm{GBq}$ for SIR-spheres $\odot$. Median stay in hospital was 4 (3 - 6) days. Median overall survival for all patients was 12.5 (95\% CI: 8.7 16.3) months.

Patients, where the tumor did not occupy more than $20 \%$ of the whole liver (estimated by the radiologist) had no different outcome compared to patients with an involvement of more than $20 \%$ of the whole liver $(\mathrm{p}=0.248)$.

Regarding the number of tumor nodules in the liver, we found no statistically significant differences in terms of overall survival in patients with one to five lesions compared to patients with more than five lesions $(\mathrm{p}=0.151)$.

The VVS showed no statistically difference in terms of survival in case of analysis of each examination separately (MRI, scintigraphy, angiography). In case of combination of all examinations a low VVS was associated with a significantly decreased survival (3.8 (95\%CI 0 - 15.0) months) compared to a medium VVS 
(10.8 (95\%CI 0 - 22.1) months), $\mathrm{p}=0.043$. For details see Table 2 and Figure 1.

Median overall survival did not differ between Theraspheres $\odot$ and SIR-spheres $\odot$. OS of patients treated with Theraspheres@ was 12.1 (95\% CI: 8.1 - 16) months and for SIR-spheres $\odot 12.5$ (95\% CI: $7.5-17.5)$ months, $\mathrm{p}=0.874$, see Figure 2 .

Table 1. Patient's characteristics. Age, laboratory: Median (interquartile range), MELD: Model of end-stage liver disease, NASH: Non-alcoholic steatohepatitis, RFA: radiofrequency ablation, AFP: alphafeto-protein.

\begin{tabular}{|c|c|}
\hline $\mathrm{n}$ & 39 \\
\hline Age (years) & $65(57-68)$ \\
\hline \multicolumn{2}{|l|}{ Sex } \\
\hline Male & $33(85 \%)$ \\
\hline Female & $6(15 \%)$ \\
\hline \multicolumn{2}{|l|}{ Child-Pugh-Score } \\
\hline $\mathrm{A}$ & 37 (95\%) \\
\hline $\mathrm{B}$ & $2(5 \%)$ \\
\hline MELD & $7(7-9)$ \\
\hline Cirrhosis & $36(92 \%)$ \\
\hline \multicolumn{2}{|l|}{ Etiology } \\
\hline Chronic hepatitis B & $13(33 \%)$ \\
\hline Alcohol & $9(23 \%)$ \\
\hline Chronic hepatitis C & $8(20.5 \%)$ \\
\hline Cryptogenic & $8(20.5 \%)$ \\
\hline NASH & $1(3 \%)$ \\
\hline \multicolumn{2}{|l|}{ BCLC-stage } \\
\hline $\mathrm{B}$ & $38(97 \%)$ \\
\hline $\mathrm{C}$ & $1(3 \%)$ \\
\hline \multicolumn{2}{|l|}{ Therapies prior SIRT } \\
\hline None & $12(31 \%)$ \\
\hline TACE & $17(44 \%)$ \\
\hline Sorafenib & $10(25.5 \%)$ \\
\hline surgery & $8(20.5 \%)$ \\
\hline RFA & $3(8 \%)$ \\
\hline \multicolumn{2}{|l|}{ Baseline laboratory } \\
\hline Leukocytes (/nl) & $6.1(4.5-7.8)$ \\
\hline Thrombocytes (/nl) & $149(102.5-187.0)$ \\
\hline Bilirubin (mg/dl) & $0.8(0.6-1.2)$ \\
\hline Albumin (g/dl) & $41.5(36.0-42.8)$ \\
\hline $\operatorname{AFP}(\mathrm{U} / \mathrm{l})$ & $135.9(66.9-1480.0)$ \\
\hline
\end{tabular}


Table 2. Prognostic factors and outcome. MRI: Magnetic resonance imaging; Overall survival: months.

\begin{tabular}{|c|c|c|c|}
\hline & $\mathbf{n}$ & Overall survival $(95 \% \mathrm{CI})$ & p-value \\
\hline Whole study cohort & 39 & $12.5(8.7-16.3)$ & \\
\hline \multicolumn{4}{|l|}{ Tumor load of the liver: } \\
\hline $1 \%-20 \%$ & 18 & $13.4(6.3-20.5)$ & \\
\hline$>20 \%$ & 21 & $10.8(8.0-13.5)$ & 0.248 \\
\hline \multicolumn{4}{|l|}{ Number of tumors: } \\
\hline $1-5$ & 15 & $16.7(7.1-26.3)$ & \\
\hline$>5$ & 24 & $10.8(7.8-13.7$ & 0.151 \\
\hline \multicolumn{4}{|c|}{ Visual vascularisation score } \\
\hline \multicolumn{4}{|l|}{ MRI } \\
\hline Low & 8 & $12.1(5.2-19.0)$ & - \\
\hline Medium & 13 & $10.8(2.8-18.7)$ & 0.698 \\
\hline High & 18 & $12.5(0.5-24.5)$ & 0.511 \\
\hline \multicolumn{4}{|l|}{ Scintigraphy } \\
\hline Low & 9 & $12.1(0-28.4)$ & - \\
\hline Medium & 15 & $9.1(6.1-12.1)$ & 0.749 \\
\hline High & 15 & $17.4(7.7-27.1)$ & 0.196 \\
\hline \multicolumn{4}{|l|}{ Angiography } \\
\hline Low & 14 & $13.4(2.3-24.5)$ & - \\
\hline Medium & 14 & $9.8(7.8-11.8)$ & 0.963 \\
\hline High & 11 & $12.5(3.0-22.0)$ & 0.415 \\
\hline \multicolumn{4}{|c|}{ Combination of all modalities } \\
\hline Low & 8 & $3.8(0-15.0)$ & - \\
\hline Medium & 19 & $10.8(0-22.1)$ & 0.043 \\
\hline High & 12 & $12.5(7.4-17.6)$ & 0.090 \\
\hline
\end{tabular}

\subsection{Adverse Events}

Grade I/II adverse events were common after SIRT.

While shortly (up to 4 days) after the procedure $36 \%$ of the patients experienced adverse events, the rate of adverse events increased to $49 \%$ two to three months after the intervention.

Abdominal pain (18\%) was the most frequent adverse event directly after SIRT but its occurrence decreased over time. Fatigue and nausea had their maximum occurrence at day 5 to 30 after SIRT, but persisted even in long-term course. Ascites was not present directly after the intervention but developed in $26 \%$ of the cases in month two and three after SIRT.

Severe adverse events were evident in 4 patients, including cholangitis, pancreatitis, anemia and renal failure occurred only in single patients respectively and at different time points. All of these four severe adverse events resolved. No treatment related Grade IV toxicity and no treatment related death was evident. For detailed specification of adverse events see Table 3. 


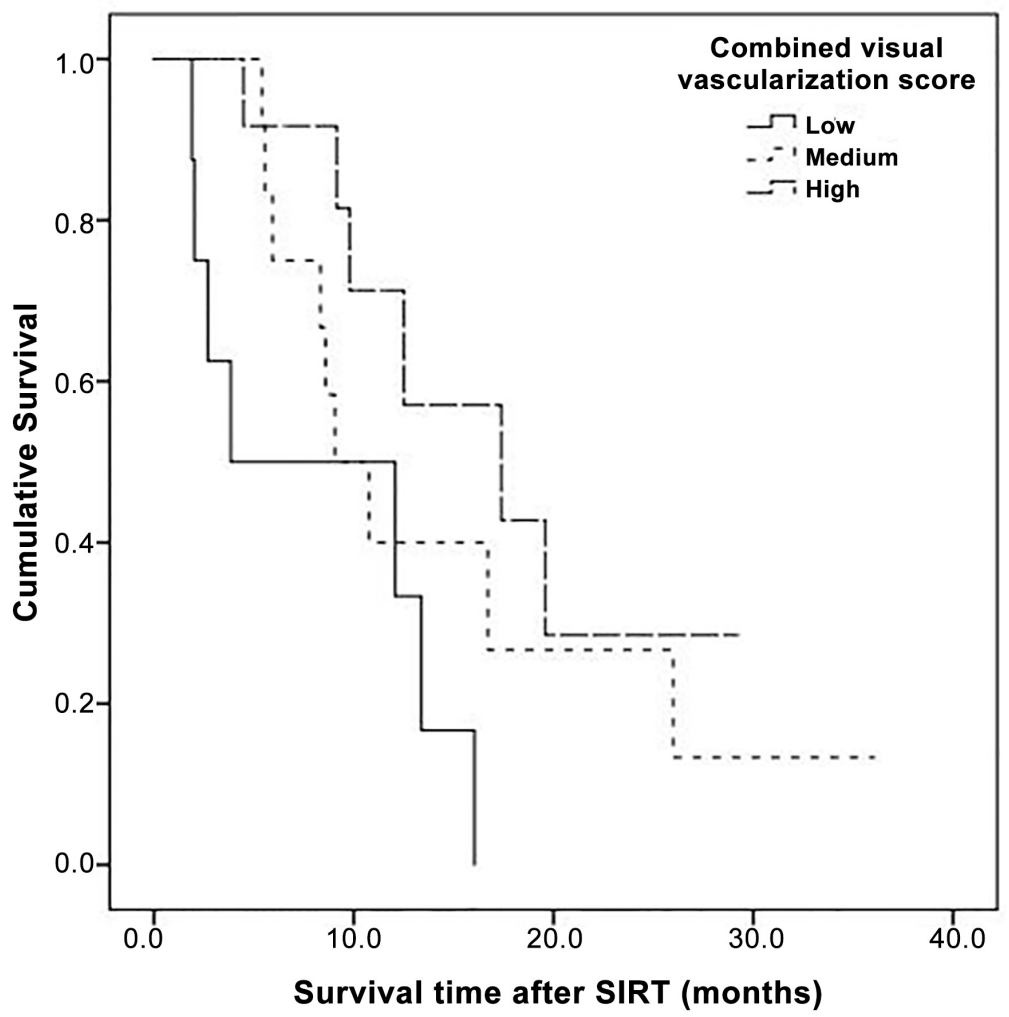

Figure 1. Survival of patients by visual vascularization score. Patients with low VVS showed significantly $(\mathrm{p}=0.043)$ decreased survival of $3.8(95 \%$ CI $0-15.0)$ months.

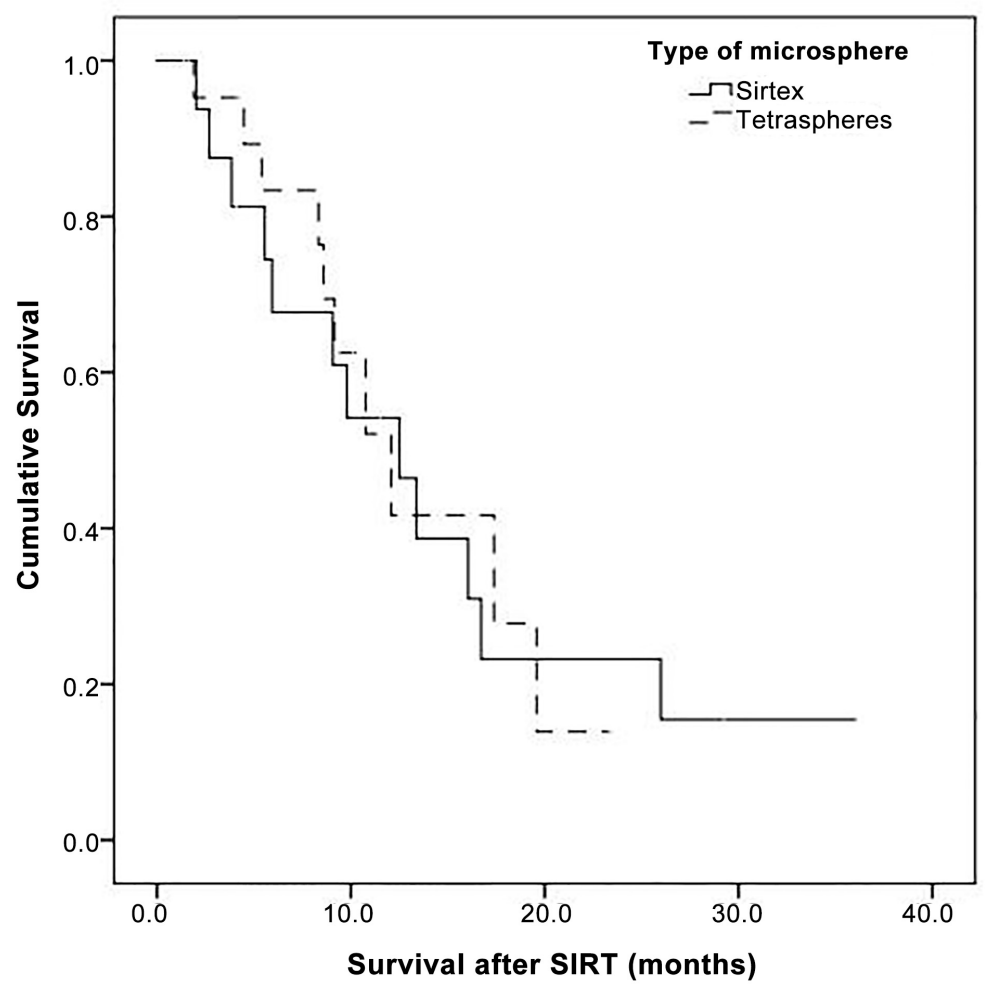

Figure 2. Comparison of patients by type of microsphere. 
Table 3. Adverse events stratified by time of onset.

\begin{tabular}{|c|c|c|c|}
\hline & Day $1-4$ & Day $5-30$ & Day $30-90$ \\
\hline Total & $14(36 \%)$ & $16(41 \%)$ & $19(49 \%)$ \\
\hline \multicolumn{4}{|l|}{ Grade I/II } \\
\hline Pain & $7(18 \%)$ & $3(8 \%)$ & $2(5 \%)$ \\
\hline Nausea & $4(10 \%)$ & $7(18 \%)$ & $5(13 \%)$ \\
\hline Fatigue & 0 & $6(15 \%)$ & $5(13 \%)$ \\
\hline Ascites & 0 & $1(3 \%)$ & $10(26 \%)$ \\
\hline Encephalopathy & 0 & $1(3 \%)$ & $2(5 \%)$ \\
\hline Fever & $2(5 \%)$ & 0 & 0 \\
\hline Hypotension & $2(5 \%)$ & 0 & 0 \\
\hline Pruritus & $1(3 \%)$ & $1(3 \%)$ & $1(3 \%)$ \\
\hline \multicolumn{4}{|l|}{ Grade III } \\
\hline Cholangitis & $1(3 \%)$ & 0 & 0 \\
\hline Pancreatitis & 0 & $1(3 \%)$ & 0 \\
\hline Renal failure & 0 & 0 & $1(3 \%)$ \\
\hline Anemia & 0 & 0 & $1(3 \%)$ \\
\hline \multicolumn{4}{|c|}{$\begin{array}{l}\text { Total treatment emergent adverse events } \\
\text { by type of microsphere: }\end{array}$} \\
\hline Theraspheres $\odot(n=23)$ & $9(39 \%)$ & $10(43 \%)$ & $12(52 \%)$ \\
\hline SIRT-Spheres@ $(n=16)$ & $5(31 \%)$ & $6(38 \%)$ & $7(44 \%)$ \\
\hline
\end{tabular}

\subsection{SIRT and Sorafenib}

Patients who received a therapy with sorafenib prior or after SIRT had a median overalls survival of 16 (95\% CI: 3.0 - 15.1) months, which was statistically longer compared to patients who did not receive sorafenib (OS: 9.1 (95\% CI: 10.3 21.8) months). When analyzing the time of sorafenib treatment in relation to time of SIRT it became evident, that sorafenib therapy after SIRT was associated with a statistically significant improved survival in comparison to sorafenib therapy before SIRT or no sorafenib therapy at all. See Figure 3 and Table 4.

\section{Discussion}

The mainstay of treatment for HCC in BCLC B stage patients is TACE [3]. However in some patients it is technically not feasible due to a lack of dedicated tumor feeding vessels, multilocular spread ( $>5$ lesions), very large lesions $(>7 \mathrm{~cm}$ diameter, which have high risk for ischemic necrosis) or major portal vein thrombosis [10]. For these patients SIRT might be an alternative treatment option, since it does not require embolization of a tumor feeding vessel. In our study 39 patients received SIRT for HCC. The toxicity of the SIRT intervention was remarkably low, with no grade 3 or 4 events reported indicating the procedure is safe for this patient cohort with a high co-morbidity due to underlying liver disease. Concerning the two commercially available spheres, it is worth to mention that both products were used according to their recommended dose calculation, respectively; this translates into remarkably different methodology. 


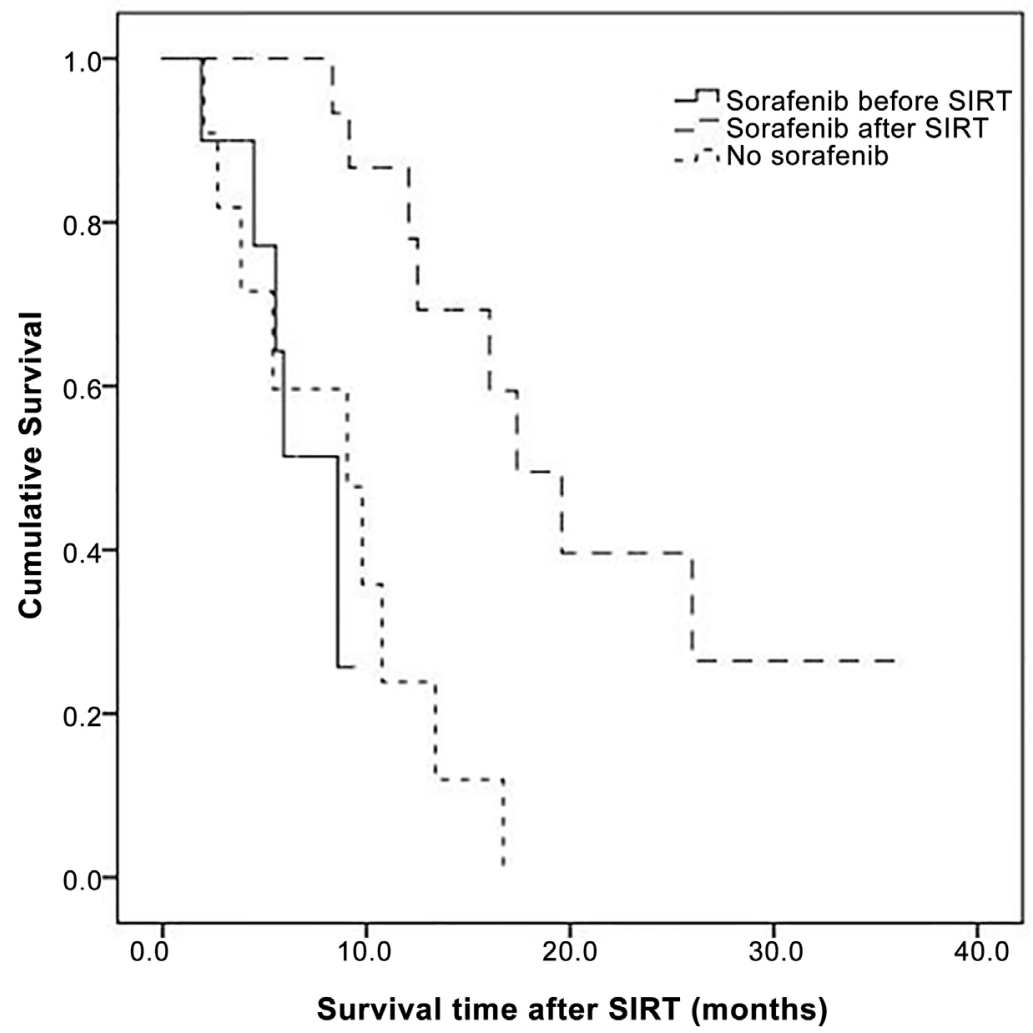

Figure 3. Survival of patients who received sorafenib after SIRT is significantly increased compared to patients who never received sorafenib $(\mathrm{p}<0.001)$ or received sorafenib before $\operatorname{SIRT}(\mathrm{p}=0.001)$.

Table 4. Overall survival (months) regarding timing of SIRT and sorafenib treatment.

\begin{tabular}{cccc}
\hline & n & Overall survival (95\% CI) & p-value \\
\hline SIRT only & 13 & $9.1(3.0-15.1)$ & $<0.001$ \\
Sorafenib before SIRT & 10 & $8.6(5.5-11.7)$ & $\mathbf{0 . 0 0 1}$ \\
Sorafenib after SIRT & 16 & $17.4(12.1-22.7)$ & - \\
\hline
\end{tabular}

In average, the radioactivity administered with Thera Spheres was 2-fold higher; in contrast the embolization effect of SIR-Spheres is increased because the single sphere is tagged with less radionuclide. Nevertheless, the respective treatment strategies chosen by the two vendors did not translate into any clinical difference, neither survival nor tolerability. This observation is well in line with previous reports comparing both products in treatment of liver metastases from mixed [7] or neuroendocrine tumor origin [8] but presents the first report demonstrating equivalent therapeutic ranges also in comparable cohorts of HCC patients complicated by concomitant liver cirrhosis.

We further looked for a correlation of the investigator assessed tumor vascularization with survival and found that a combined score of established visual vascularization techniques including MAA-scintigraphy, MRI and angiography correlated with survival after SIRT. Similar findings regarding the degree of 
vascularization and response to treatment have been reported for TACE in HCC patients [11]. The number of tumors nodules and the tumor load in percentage of liver tissue, however, did not significantly correlate with survival. This interesting finding for SIRT differs from reported predictors of TACE efficacy. Here tumor load appears to be a relevant factor predicting survival [12]. For SIRT, a recently published prognosis score [13] found that MELD and CRP levels determined four weeks after SIRT correlated with survival. In our cohort MELD and CRP were determined at baseline and those did not correlate with overall survival.

We further analyzed those patients who received sorafenib prior to SIRT and those treated with sorafenib after SIRT compared to patients who never received sorafenib. Interestingly, patients who received sorafenib initially, then SIRT upon progression responded similarly as patients who never received sorafenib, indicating that sorafenib treatment is still feasible after SIRT. This is in line with the mode of action of sorafenib which appears to have mainly normalization effects on the architecture of tumor vessels rather than inhibiting vascularization perse [14]. Further, patients who received sorafenib having progressed after SIRT had been performed had a markedly increased survival over the other cohorts. This indicates that the application of SIRT does not interfere with the anti-angiogenic activity of sorafenib making this a plausible therapeutic sequence that showed an additive effect on overall survival. In contrast, for TACE followed by sorafenib Ha et al. could not show additive activity of TACE and sorafenib which may be due to the embolization of tumor vessels, the main target of sorafenib; by TACE but not by SIRT [15]. Limitation of our study is the small patient number and its retrospective nature. Hence, the data should be interpreted cautiously. A larger prospective trial on therapeutic sequencing is clearly warranted.

In conclusion, SIRT is safe and of low toxicity in HCC patients. No differences in toxicity and outcome are observed between the different spheres. Finally, the sequential systemic therapy with sorafenib appears to be additive in regard to survival after SIRT.

\section{References}

[1] Llovet, J.M., Bru, C. and Bruix, J. (1999) Prognosis of Hepatocellular Carcinoma: The BCLC Staging Classification. Seminars in Liver Disease, 19, 329-338.

https://doi.org/10.1055/s-2007-1007122

[2] Hsu, C.Y., Huang, Y.H., Chiou, Y.Y., Su, C.W., Lin, H.C., Lee, R.C., Chiang, J.H., Huo, T.I., Lee, F.Y. and Lee, S.D. (2011) Comparison of Radiofrequency Ablation and Transarterial Chemoembolization for Hepatocellular Carcinoma within the Milan Criteria: A Propensity Score Analysis. Liver Transplantation, 17, 556-566. https://doi.org/10.1002/lt.22273

[3] Lee, Y.B., Lee, D.H., Cho, Y., Yu, S.J., Lee, J.H., Yoon, J.H., Lee, H.S., Kim, H.C., Yi, N.J., Lee, K.W., et al. (2015) Comparison of Transarterial Chemoembolization and Hepatic Resection for Large Solitary Hepatocellular Carcinoma: A Propensity Score Analysis. Journal of Vascular and Interventional Radiology, 26, 651-659. https://doi.org/10.1016/j.jvir.2015.02.004 
[4] Burrel, M., Reig, M., Forner, A., Barrufet, M., de Lope, C.R., Tremosini, S., Ayuso, C., Llovet, J.M., Real, M.I. and Bruix, J. (2012) Survival of Patients with Hepatocellular Carcinoma Treated by Transarterial Chemoembolisation (TACE) Using Drug Eluting Beads. Implications for Clinical Practice and Trial Design. Journal of Hepatology, 56, 1330-1335. https://doi.org/10.1016/j.jhep.2012.01.008

[5] Llovet, J.M., Ricci, S., Mazzaferro, V., Hilgard, P., Gane, E., Blanc, J.F., de Oliveira, A.C., Santoro, A., Raoul, J.L., Forner, A., et al. (2008) Sorafenib in Advanced Hepatocellular Carcinoma. The New England Journal of Medicine, 359, 378-390. https://doi.org/10.1056/NEJMoa0708857

[6] Allison, C. (2007) Yttrium-90 Microspheres (TheraSphere and SIR-Spheres) for the Treatment of Unresectable Hepatocellular Carcinoma. Issues in Emerging Health Technologies, No. 102, 1-6.

[7] Wong, C.Y., Savin, M., Sherpa, K.M., Qing, F., Campbell, J., Gates, V.L., Lewandowski, R.J., Cheng, V., Thie, J., Fink-Bennett, D., et al. (2006) Regional Yttrium-90 Microsphere Treatment of Surgically Unresectable and Chemotherapy-Refractory Metastatic Liver Carcinoma. Cancer Biotherapy \& Radiopharmaceuticals, 21, $305-$ 313. https://doi.org/10.1089/cbr.2006.21.305

[8] Rhee, T.K., Lewandowski, R.J., Liu, D.M., Mulcahy, M.F., Takahashi, G., Hansen, P.D., Benson3rd, A.B., Kennedy, A.S., Omary, R.A. and Salem, R. (2008) 90Y Radioembolization for Metastatic Neuroendocrine Liver Tumors: Preliminary Results from a Multi-Institutional Experience. Annals of Surgery, 247, 1029-1035. https://doi.org/10.1097/SLA.0b013e3181728a45

[9] Mosconi, C., Cappelli, A., Pettinato, C. and Golfieri, R. (2015) Radioembolization with Yttrium-90 Microspheres in Hepatocellular Carcinoma: Role and Perspectives. World Journal of Hepatology, 7, 738-752. https://doi.org/10.4254/wjh.v7.i5.738

[10] Van Ha, T.G. (2009) Transarterial Chemoembolization for Hepatocellular Carcinoma. Seminars in Interventional Radiology, 26, 270-275. https://doi.org/10.1055/s-0029-1225672

[11] Sergio, A., Cristofori, C., Cardin, R., Pivetta, G., Ragazzi, R., Baldan, A., Girardi, L., Cillo, U., Burra, P., Giacomin, A., et al. (2008) Transcatheter Arterial Chemoembolization (TACE) in Hepatocellular Carcinoma (HCC): The Role of Angiogenesis and Invasiveness. The American Journal of Gastroenterology, 103, 914-921. https://doi.org/10.1111/j.1572-0241.2007.01712.x

[12] White, J.A., Redden, D.T., Bryant, M.K., Dorn, D., Saddekni, S., Abdel Aal, A.K., Zarzour, J., Bolus, D., Smith, J.K., Gray, S., et al. (2014) Predictors of Repeat Transarterial Chemoembolization in the Treatment of Hepatocellular Carcinoma. $H P B$, 16, 1095-1101. https://doi.org/10.1111/hpb.12313

[13] Weng, Z., Ertle, J., Zheng, S., Lauenstein, T., Mueller, S., Bockisch, A., Gerken, G., Yang, D. and Schlaak, J.F. (2013) A New Model to Estimate Prognosis in Patients with Hepatocellular Carcinoma after Yttrium-90 Radioembolization. PLoS ONE, 8 , e82225. https://doi.org/10.1371/journal.pone.0082225

[14] Shiozawa, K., Watanabe, M., Ikehara, T., Kogame, M., Kikuchi, Y., Igarashi, Y. and Sumino, Y. (2016) Therapeutic Evaluation of Sorafenib for Hepatocellular Carcinoma Using Contrast-Enhanced Ultrasonography: Preliminary Result. Oncology Letters, 12, 579-584. https://doi.org/10.3892/ol.2016.4669

[15] Ha, Y., Lee, D., Shim, J.H., Lim, Y.S., Lee, H.C., Chung, Y.H., Lee, Y.S., Park, S.R., Ryu, M.H., Ryoo, B.Y., et al. (2016) Role of Transarterial Chemoembolization in Relation with Sorafenib for Patients with Advanced Hepatocellular Carcinoma. Oncotarget, 7, 74303-74313. https://doi.org/10.18632/oncotarget.11030 
Submit or recommend next manuscript to SCIRP and we will provide best service for you:

Accepting pre-submission inquiries through Email, Facebook, LinkedIn, Twitter, etc. A wide selection of journals (inclusive of 9 subjects, more than 200 journals)

Providing 24-hour high-quality service

User-friendly online submission system

Fair and swift peer-review system

Efficient typesetting and proofreading procedure

Display of the result of downloads and visits, as well as the number of cited articles Maximum dissemination of your research work

Submit your manuscript at: http://papersubmission.scirp.org/

Or contact jet@scirp.org 